\title{
The influence of concurrent load on mouthed and vocalized modality effects
}

\author{
JUDY CANTOR and RANDALL W. ENGLE \\ University of South Carolina, Columbia, South Carolina
}

\begin{abstract}
A visual search task was coupled with the serial recall of words to assess the extent to which modality effects mediated by vocalizing and silent mouthing reflect an automatically activated preattentive process. Overall, serial position functions systematically changed as concurrent task demands increased, but the magnitude of the modality effect associated with both mouthing and vocalizing was not altered, regardless of whether or not subjects simultaneously searched for digits. These results support the notion that modality effects index a preattentive process that can be activated automatically by either spoken input or gestural cues associated with speech.
\end{abstract}

Sensory registers have been implicated as basic structural features of the human information processing system and viewed as passive repositories that briefly represent modality-specific primitive sensory information (Atkinson \& Shiffrin, 1968). For the auditory mode, Neisser (1967) postulated the existence of an echoic memory that temporarily preserves individual auditory sound segments and enables the determination of complete sound patterns.

Crowder and Morton (1969) formalized the first comprehensive theory of echoic memory, which was based on the finding that the auditory presentation of a series of verbal items leads to better recency performance than does visual presentation (Conrad \& Hull, 1968; Corballis, 1966; Murray, 1966). They suggested that this modality effect is mediated by a precategorical acoustic storage (PAS) system that maintains the sensory features of auditory input for several seconds, enabling a comparison between traces persisting in PAS and representations in short-term memory. They further demonstrated that when an additional not-to-be-remembered auditory item (i.e., a suffix) follows the terminal list item, the modality effect is eliminated, suggesting that PAS is sensitive to masking and thereby limited to the retention of the final unmasked item.

The present article concerns two of the properties that Crowder and Morton (1969) attributed to PAS. First, as the initial step in information processing, PAS was conceptualized as precategorical, thereby preceding the extraction of meaning and item identification on any level higher than simple physical-feature analysis. As a corollary, PAS was viewed as an automatically activated preattentive store. Second, it was posited that PAS must be

This study was part of a master's thesis submitted to the University of South Carolina by Judy Cantor. Special thanks are extended to Dan Fisk for his help in conceptualizing the dual-task analysis and Julie Carullo for testing subjects. The research was supported by AFOSR Grant 870069 to Randall W. Engle. Reprints may be obtained from Judy Cantor, Department of Psychology, University of South Carolina, Columbia, SC 29208. mediated by spoken input, be it active vocalization or passive listening, since these conditions produce both modality and suffix effects, whereas silent reading does not (Conrad \& Hull, 1968; Murray, 1966).

Evidence for a precategorical store was provided by Morton, Crowder, and Prussin (1971), who reported that suffix effects vary only with changes in the physical features of the stimulus material. Thus, manipulating the taxonomic relationship between list items and suffixes does not change the suffix effect, whereas this effect is reduced when the suffix and list items are presented in different pitches, by different speakers, or to different ears. Although these findings support the notion that suffix effects reflect a precategorical mechanism, they do not provide evidence for the corollary to this assumption, that the process underlying these effects are preattentive.

Greenberg and Engle (1983) directly tested the preattentive nature of PAS by requiring subjects to attend to different suffixes that signaled either recall or nonrecall. If the suffix effect reflects an attentional mechanism, then increasing attention to a suffix should increase its effect. Greenberg and Engle found, however, that forcing attention to the suffix increased the suffix effect for preterminal items, but had little effect on terminal items. Converging evidence was found by Balota and Engle (1981), who reported that the suffix effect associated with the final item of auditory lists was not altered by the manipulation of presentation rate or practice. These results support the notion that attentional, strategic factors do not underlie auditory suffix effects, and uphold Crowder and Morton's (1969) proposal that these effects are mediated by a preattentive store.

Another characteristic that Crowder and Morton (1969) attributed to their version of echoic memory is that representation in PAS must be mediated by spoken input, since listening to auditorily presented lists and vocalizing visually presented items were the only conditions under which modality and suffix effects were obtained (Conrad \& Hull, 1968; Murray, 1966). However, subsequent studies have reported modality and suffix ef- 
fects when subjects read the lips of a silent experimenter (Campbell \& Dodd, 1980; Spoehr \& Corin, 1978) and when visually presented stimuli are silently mouthed (Green \& Crowder, 1984, 1986; Nairne \& Crowder, 1982; Nairne \& Walters, 1983). Clearly, these findings conflict with the original notion that PAS represents only overt speech.

To account for nonauditory modality and suffix effects, an updated version of PAS now incorporates silent speech gestures into the auditory sensory system (Crowder, 1983; Greene \& Crowder, 1984). In the revised model, processing can be activated by nonauditory cues such as mouthing and lipreading, producing representations in PAS equivalent to those of audition. The result is "functional sound" (Crowder, 1983, p. 261). While this newer PAS model can account for similarities in performance between mouthed and spoken lists, several differences remain to be fully explained. For example, one finding typically associated with auditory presentation is a decrease in recency when stimuli are phonologically confusable (Crowder, 1971, 1973; Watkins, Watkins, \& Crowder, 1974). But reports associated with mouthed stimuli are inconclusive. Although Greene and Crowder (1984) demonstrated that acoustically similar stimuli reduce suffix effects with mouthing, Turner et al. (1987) found that the suffix effect produced by mouthing did not depend on the phonological nature of the stimulus material. In addition, recall associated with the final items of mouthed lists is often poorer than that associated with vocalized lists (cf. Green \& Crowder, 1984, 1986; Nairne \& Walters, 1983), and mouthed suffixes following spoken lists produce smaller suffix effects than when list items and suffixes are presented in the same mode (Greene \& Crowder, 1984).

Greene and Crowder (1984) attribute the differences in performance on mouthed as opposed to spoken stimuli to the structural characteristics of PAS, suggesting that mouthed and auditory stimulation may be represented on different channels of the system, or that mouthed items may be perceptually more difficult for PAS to disambiguate than spoken items. There is, however, an alternative explanation, in which modality and suffix effects for mouthing reflect attentional working-memory processes rather than a preattentive echoic memory. While there is evidence that suffix effects produced with spoken input reflect a preattentive mechanism, it is yet to be determined whether mouthing effects are similarly preattentive.

One possibility is that mouthing reduces the ability for covert rehearsal, thereby placing high demands on working memory. Two results follow: The total number of items that can be retained is reduced, and the most recently mouthed items have the greatest likelihood of still being in working memory at the time of recall. Mouthing should produce the poorest overall performance (c.f. Greene \& Crowder, 1984, 1986; Nairne \& Walters, 1983), and the items that are retained tend to be those near the end of the list. This contrasts with silently read lists when rehearsal is not prevented. When lists are read silently, rehearsal of early items is not suppressed. Hence, the most frequently rehearsed items are more likely to be represented in working memory, and are most likely to be recalled. The result is high primacy and little recency. In addition, Crowder and Morton (1969) postulated that PAS analysis and higher level encoding proceed simultaneously. Thus all items, regardless of presentation modality, are represented in working memory. Given the similarities between overt speech and silent mouthing, the small suffix effect found when a mouthed suffix follows a vocalized list may simply indicate interference in working-memory processes rather than interference in PAS.

The current study was designed to test whether modality effects produced by mouthing and by vocalizing reflect the same mechanism, and whether the processes underlying these effects are preattentive. A dual-task paradigm was adopted, in which the primary task was the serial recall of words, and the secondary task consisted of a choice reaction time, varied-mapping visual digit search with two levels of complexity. For the single search task, the subjects were presented with a memory set of one or three digits, followed by eight different test frames of four digits each. During the presentation of each test frame, the task was to decide whether or not any of the memoryset digits were displayed. This type of visual search task has been previously shown to require attention and resources from a limited-capacity system (Schneider \& Shiffrin, 1977; Shiffrin \& Schneider, 1977). For the single recall task, the subjects mouthed, vocalized, or silently read a series of words, and then attempted serial recall. This task most closely resembles the typical procedure in which modality effects are found. In the dual task, a to-be-remembered word was presented with each test frame of the digit search. The subjects performed the digit search while simultaneously mouthing, vocalizing, or silently reading words, which were to be recalled at the end of each trial.

According to the PAS model, when stimuli are spoken or mouthed, performance is mediated by the abilities to draw on working-memory capacity and to rely on capacity-independent echoic memory processes. The persistent traces in echoic memory produce the advantage of spoken or mouthed input over silent reading in the final serial position. To the extent that a concurrent task reduces a subject's ability to rely on working memory, a remaining advantage may reflect a preattentive echoic memory. Support for this conclusion would be demonstrated if modality effects associated with vocalizing and mouthing can be obtained under both single- and dualtask conditions and if the magnitude of these effects does not differ between tasks. Whereas reducing the ability of subjects to use working memory should produce decrements in overall recall for all presentation modalities, an advantage of spoken or mouthed input over silent reading in the final serial position should remain constant. In addition, if, as Greene and Crowder (1984) speculated, the superiority of spoken input over silent mouthing reflects the structural characteristics of echoic memory, then we 
predict that the magnitude of this difference should also remain stable under concurrent load conditions.

In contrast, if modality effects for vocalization and mouthing reflect different underlying processes, then an alternative pattern of results would be predicted. In the single recall task, modality effects associated with both mouthing and vocalizing would be expected, replicating the results of previously reported work. Modality effects associated with vocalizing are still posited to reflect echoic memory, and they should therefore show little change as a function of concurrent load. However, if mouthed recency reflects working memory, and if mouthing reduces covert rehearsal in working memory, then the addition of a concurrent task that also requires resources should have its greatest impact on mouthed lists. Recency associated with mouthing should be significantly reduced, and the effect of concurrent load on these lists should more closely resemble that of silently read lists, thereby diverging from effects associated with vocalizing. Thus, with increases in concurrent load, the modality effect produced by mouthing should decrease, and the difference in performance on the final serial position of mouthed versus vocalized lists should increase.

\section{METHOD}

\section{Subjects}

Thirty undergraduate students from the University of South Carolina volunteered to participate for $1 \mathrm{~h}$ a day over 3 days of testing, to fulfill a course requirement or receive extra credit.

\section{Materials and Apparatus}

A pool of 1,000 high-frequency 4-6 letter nouns was constructed from Francis and Kučera's (1982) word norms. Although the same pool of words was randomly sampled on each day of testing, the order of words differed between days, and the same word did not appear more than once per day. All stimuli were presented with an IBM XT, on an Amdek monochrome monitor, with visual angle no greater than $2^{\circ}$.

\section{Single Search Task}

Trials in the single search task contained several frames of stimuli. Their sequencing is represented in Figure 1.

Memory-set frame. The first frame displayed a memory set of either one or three digits centered on the screen. All digits were randomly selected from the range $0-9$, with the constraints that no digit appear more than once in a single memory set and that all digits appear approximately an equal number of times as memoryset items. The subjects subsequently searched for these memoryset items during the presentation of stimulus frames. The level of difficulty for this task was determined by the number of items for which the subjects were to search (one or three). The memory-set frame was displayed for $2 \mathrm{sec}$.

Orienting frame. Coincident with the offset of the memory-set frame was the presentation of a frame of Xs displayed for $2 \mathrm{sec}$. The placement of these Xs corresponded to the same coordinates as the items in the stimulus frames that followed. The examples provided in Figure 1 exaggerate the acutal frame sizes. The computer screen measured $9 \times 7$ in., and the perimeter of the $X s$ and subsequent stimulus materials measured $1.7 \times 0.75$ in.

Stimulus frames. The orienting frame was followed by eight different stimulus frames, each consisting of a word centered on the screen, and cornered by four digits. These items appeared in
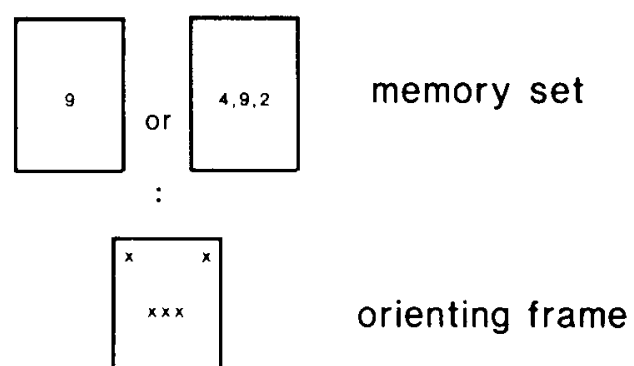

$x$
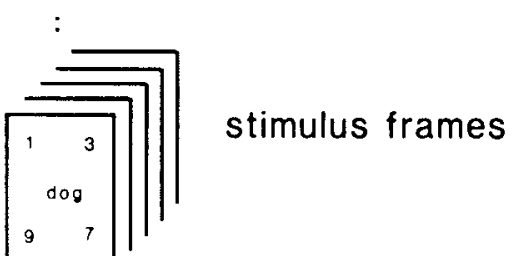

frames
: search response

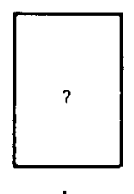

recall delay

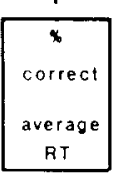

feedback frame

Figure 1. Sequencing of events for a single trial of the single search task.

the same locations as did the Xs in the orienting frame. All digits appeared approximately an equal number of times in each of the four possible positions, and no digit appeared more than once per frame. The eight stimulus frames were presented in succession, at a rate of one every $2 \mathrm{sec}$. At the time of presentation, and for each stimulus frame, the subjects were required to depress one of two buttons on an IBM mouse, indicating whether at least one of the memory-set digits appeared in the corresponding stimulus frame. Reaction times and subjects' "yes"/"no" responses were recorded. For each trial, there was an equal number of randomly ordered positive ("yes") and negative ("no") frames.

The design for this task follows the commonly used variedmapping procedure outlined by Schneider and Shiffrin (1977; see also Shiffrin \& Schneider, 1977). All digits were equally represented as memory-set items and as targets and distractors in the stimulus frames, so that the subjects were unable to predict which stimuli to target or focus on for subsequent responding. This lack of consistent mapping has been shown to prevent the development of automatic processes that can be used to perform the task, regardless of the number of trials administered.

Feedback frames. Following the final stimulus frame, the subject's average response time and percent of correct "yes"/ "no" answers was displayed for $2 \mathrm{sec}$. The subjects were encouraged to respond as quickly and accurately as possible. The offset of the feedback frame was followed by a new memory set, indicating the beginning of a new trial.

For the single search task, two blocks of 12 trials each were presented on Day 1, and two blocks of 16 trials were presented on Days 2 and 3. For each block, half of the trials contained a memory set of one item, and the remainder had three items in the 
memory set. The order of presentation for these trials was randomly selected.

\section{Single Recall Task}

For this task, the general pattern of display frames was similar to that of the single search task. However, for the single recall task, the memory-set frame was replaced by a frame of three Xs centered on the screen. The eight stimulus frames had the same construction as those in the single search task: A word was centered on the screen, and surrounded by four digits. For this task, however, the subjects were to ignore the digits. In addition, the feedback frame was omitted, and a recall period was inserted following the final stimulus frame, and indicated by the 28-sec delay of a single question mark. The end of the recall period was marked by a brief $800 \mathrm{~Hz}$ tone, which cued subjects that a new trial was to begin. The recall period was to be used for immediate written serial recall in the appropriate response booklets. Unlike the single search task, the single recall task did not vary in difficulty. Thus the total number of trials for this task was half the number for the single search. Two blocks of six trials each were presented on Day 1, and two blocks of eight trials each on Days 2 and 3.

\section{Dual Task}

The dual task can be considered a combination of the two previously described single tasks. The sequencing of display frames was again similar to that depicted in Figure 1. A memory set of one or three items was presented, followed by an orienting frame, the eight stimulus frames, and the subject's search response. However, preceding the feedback frame was the presentation of a question mark for $28 \mathrm{sec}$, during which the subjects were to serially recall the words. In addition, prior to the onset of a block of dual-task trials, the overall average reaction time and percent of correct "yes" "/ "no" responses for the most recently presented single search block was displayed. The subjects were not aware that these numbers reflected their previous single search performances but were simply told to try to maintain the performance displayed throughout the block of dual-task trials. The number of trials for the dual task was the same as for the single search task. Response booklets for immediate written serial recall were provided.

\section{Procedure}

All subjects participated for $1 \mathrm{~h}$ a day over 3 days of testing. The subjects were randomly assigned to one of three modality conditions, and this assignment did not change over the testing days. Accordingly, the subjects were instructed to vocalize, mouth, or silently read the word in each stimulus frame. An experimenter remained in the testing room to monitor adherence to the instructions.

As previously mentioned, performance on the single search task was used as a baseline for subjects to maintain during the dual task. However, for the subjects assigned to the mouthing and vocalizing conditions, reaction times to the dual task reflected both visual search time and the time required to mouth or vocalize words. To examine the reaction times in the dual task in relation to the single search reaction times, it was necessary to account for the time required to vocalize or mouth words. It was therefore decided that the subjects in these conditions would also mouth or vocalize words during the single search task. The critical difference between these tasks remained. For the dual task, the subjects had to perform the digit search while simultaneously attempting to remember all of the words presented in a trial. In contrast for the single search task, the subjects had to vocalize or mouth words while performing the search, but they did not have to remember them.

Using the measures from the single search task as a baseline for the dual task placed an additional constraint on the order in which tasks could be administered. Because the subjects were to maintain their reaction times and error rate performances at the same levels as on the most recently presented block of single search trials, a block of single search would necessarily have to precede each block of the dual task. Therefore, a task order was chosen that remained constant throughout the 3 days of testing. On each day, the subjects were presented with a block of single search trials, followed by a block of single recall trials, and then a block of the dual task. Following a 10 -min break, the task order was repeated. Thus each task was performed twice on each day.

For the single search, the subjects were instructed to perform as quickly and accurately as possible, and the subjects in the mouthing and vocalizing conditions were told that once they had performed this manipulation they should not attempt to remember the words. For the single recall task, all subjects were instructed to ignore the digits in the stimulus frames and to concentrate on remembering the words in the order in which they were presented. Notice that this task most closely resembles the typical modality-effect task. Instructions for the dual task emphasized that the subjects should maintain both percent correct and reaction times for the search task, while simultaneously attempting to remember words in the order presented.

\section{RESULTS}

Separate analyses were computed on the recall, reaction time, and error rate scores, and all post hoc comparisons were computed with the Tukey HSD. Where statistically significant effects are reported, alpha levels are at .05 or better. Day 1 of testing was used to familiarize subjects with the task requirements. Hence only the data for Days 2 and 3 were included in the analyses.

\section{Word Recall}

Items were scored correct if they were recalled in the same serial position in which they were presented. The recall data were submitted to a 3 (modality) $\times 2$ (day) $\times$ 3 (task) $\times 8$ (serial position) mixed design analysis of variance (ANOVA), reflecting the between-subjects factor of modality (vocalized, mouthed, or silently read words), and the within-subjects factors of day (Day 2 or 3 of testing), task (single recall, dual task with a memory-set size of one, and dual task with Set Size 3), and serials position (one through eight).

Figure 2 shows the percent of correct responses as a function of serial position, modality, and task type collapsing across Days 2 and 3. Although overall performance improved from Day 2 to Day $3[F(1,27)=5.87]$, separate functions for each day of testing are not presented, since day did not interact with any of the other factors. As is shown in Figure 2, clear modality effects associated with vocalizing and mouthing were obtained in the single task and for both levels of the dual task. These effects were statistically confirmed by the modality $\times$ task $X$ serial position interaction $\left[F(28,378)=3.08, M S_{\mathrm{e}}=\right.$ 4.98]. Post hoc analyses indicated an advantage of vocalizing over silent reading in Serial Positions 6, 7, and 8 for all tasks. Mouthing produced better performance than did silent reading in Serial Postions 7 and 8 in the single task, and 6, 7, and 8 for both levels of the dual task. These results support previous findings that the modality effect can be mediated by both spoken and gestural input. 
Single

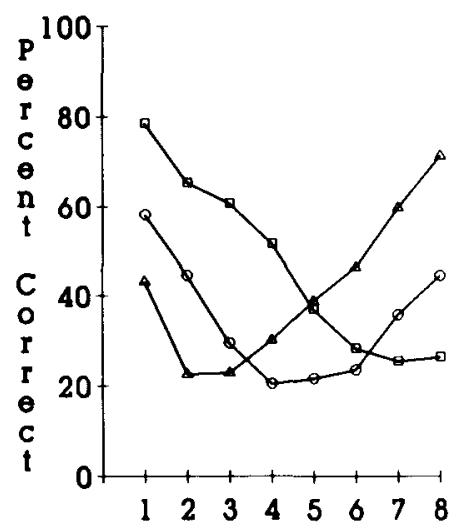

Dual

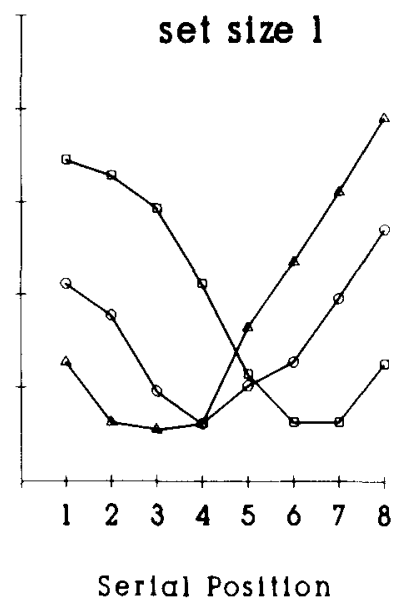

\section{Dual}

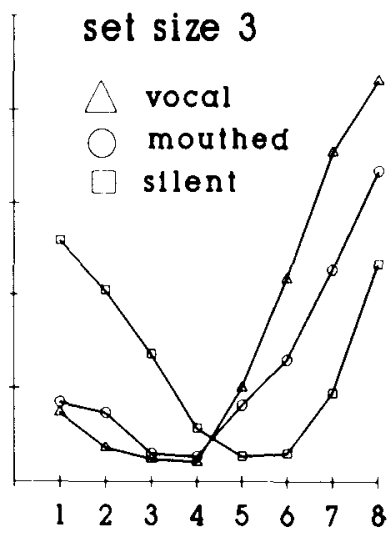

Figure 2. Percent of correctly recalled items as a function of modality of presentation serial position, and level of concurrent load.

The analysis also yielded several other significant results. For all modalities of presentation, performance in the single task was superior to both levels of the dual task, and recall was greater when the memory-set size was one than when set size was three $\left[F(2,54)=62.68, M S_{\mathrm{e}}=\right.$ 9.20 ]. Thus, recall decreased as concurrent load increased. These results support the notion that the ability to retain words depends on the same limited-capacity system required to search for digits. In addition, the interaction of task and modality indicated that when the memoryset size was three, performance on silently read lists dropped significantly more than that on mouthed and vocalized lists $\left[F(4,54)=4.57, M S_{\mathrm{e}}=9.20\right]$. The analysis yielded other significant findings, including a main effect for serial position $\left[F(7,189)=20.95, M S_{\mathrm{e}}=\right.$ 30.51], and significant interactions of modality $\times$ serial position $\left[F(14,189)=18.59, M S_{\mathrm{e}}=30.50\right]$ and task $\times$ serial position $\left[F(14,378)=25.99, M S_{e}=4.98\right]$. No other effects were statistically significant.

The recall analysis indicated clear modality effects in all tasks, and one of the questions motivating this study was whether the magnitude of the modality effect is influenced by the attentional demands of a concurrent task. There were systematic decreases in performance as concurrent task demands increased, indicating that overall performance on the recall task was constrained by a limited-capacity system. The PAS model predicts that the modality effect does not reflect such a system and should therefore not be influenced by concurrent load. With the modality effect defined as the advantage of spoken or mouthed input over silent reading in the final list position, we assessed the influence of concurrent load on the modality effect by submitting the recall performance on the final serial position to a modality $\times$ task mixed ANOVA. The results indicated that the relationship between modalities in the terminal position did not change as a function of task. Thus, the interaction of modality $\times$ task was not significant
$\left[F(4,54)=1.12, M S_{\mathrm{c}}=14.29\right]$. These results indicate that the magnitude of the modality effect is not influenced by concurrent task demands, supporting the contention that modality effects may not be mediated by a limited-capacity attentional system.

However, other explanations remain for the stability of the modality. As can be seen in Figure 2, concurrent load appears to have systematically affected performance for all modalities in the same way. Increases in processes demands corresponded to increases in recency and decrements in primacy. Thus, the stability of the modality effect may simply reflect a primacy-recency tradeoff. In dual-task conditions, subjects are required to learn a memory set while at the same time attempting to encode and remember words. Because the memory set is critical for performing the search task, the subjects may emphasize learning these items at the beginning of each trial, and abandon words presented at the same time. The larger the memory set, the more the time required to learn it, and the less the emphasis on words. Hence, primacy decreases, because the subjects attend more to the search task at the beginning of each trial. As the difficulty of this task increases, primacy decreases. Why, then, does recency improve? One possibility is that when the memory set is learned, more resources can be allocated to the words. Because primacy items are not well encoded, these additional resources are dedicated to the end of the list, and recency improves. Another possibility is enhanced recency reflects a reduction in output interference. If primacy items are not well encoded, then they impose little interference during recall. This reduction in output interference would then result in recency improvements. Primacy is still posited to reflect a limited-capacity system, but increases in recency are a function of reduced output interference. Unfortunately, our study was not designed to test the different explanations for the primacy-recency tradeoff. Nonetheless, if the improve- 
ments in recency seen in all modalities occur because available working-memory resources increase, or because output interference decreases, then the stability of the modality effect cannot be attributed to echoic memory.

If performance in all modalities reflects a primacyrecency tradeoff, then as primacy between modalities becomes similar, recency performance should also converge. In the final panel of Figure 2, where primacy overlaps for mouthed and vocalized conditions, recency should similarly overlap. Unfortunately, primacy for these conditions is at a floor, so any differences between these modalities cannot be detected. In an attempt to further explore the primacy-recency tradeoff explanation of our modality effects, the data were reanalyzed for subjects whose performance on the first two items of the dual task was above $15 \%$ correct. Omitted were the data for 5 subjects in both the mouthed and vocalized conditions, and 1 subject in the silent condition.

Two predictions follow from the primacy-recency tradeoff explanation of our modality effects. First, since we have chosen only the subjects with high primacy, their recency, and more important, their performance on the final item should be lower than that shown in the final panel of Figure 2. In addition, to the extent that primacy between mouthed and vocalized conditions still converges, final item performance should also be similar. The results are presented in Figure 3. Primacy for mouthed and vocalized conditions still converges, but no such effect is found for the terminal list item. In fact, there is no difference in absolute performance on the final item of mouthed and vocalized lists between Figures 2 and 3 . This is not the case for silently read lists. The removal of a single subject, who showed no primacy, changed the serial position function such that performance on the final item of silently read lists is significantly lower in the final panel of Figure 3 than in Figure 2. Performance on the final item of silently read lists shows the influence of a primacyrecency tradeoff, while performance on the same item for mouthed and vocalized lists shows no such influence. Although it is clear that increasing load produced a tradeoff for all modalities, it also appears that this cannot be used to explain our modality effects.

It is still possible to explain our results without reference to echoic memory. Recency may simply improve over time with practice, and be independent of primacy. Because a constant task order was chosen for this experiment, both single tasks always preceded the dual task, so that the subjects had ample practice prior to performing the dual task. If recency simply increases over time with practice, then performance on the final list item in all conditions would improve from single- to dual-task trials, and the modality effect would remain stable. There are several reasons to reject this line of thought: First, level of difficulty was manipulated randomly within blocks of dual-task trials, so there would be no reason to expect differences in recency between levels of the dual task. Furthermore, if recency is a function of practice rather task difficulty, then when the data for each task are broken down by days and blocks, recency should increase as practice increases. As indicated earlier, overall performance did improve from the second to the third day of testing, but this factor did not interact with any other factors, including serial position $[F(7,189)=1.07]$. In addition, when the recall analysis was recomputed to include the factor of block, performance did improve over blocks, but block did not interact with any other factors, including serial position $[F(7,189)=1.48]$, and day of testing $[F(1,27)<1]$. These results do not support the notion that the stability of our modality effects were mediated by practice effects.

It is still possible that the processing requirements of performing the search task and those that produce modality effects both rely on a limited working memory, rather than a preattentive store. Thus, subjects could not perform these tasks simultaneously when the resource demands exceed capacity. The result would be a decreased
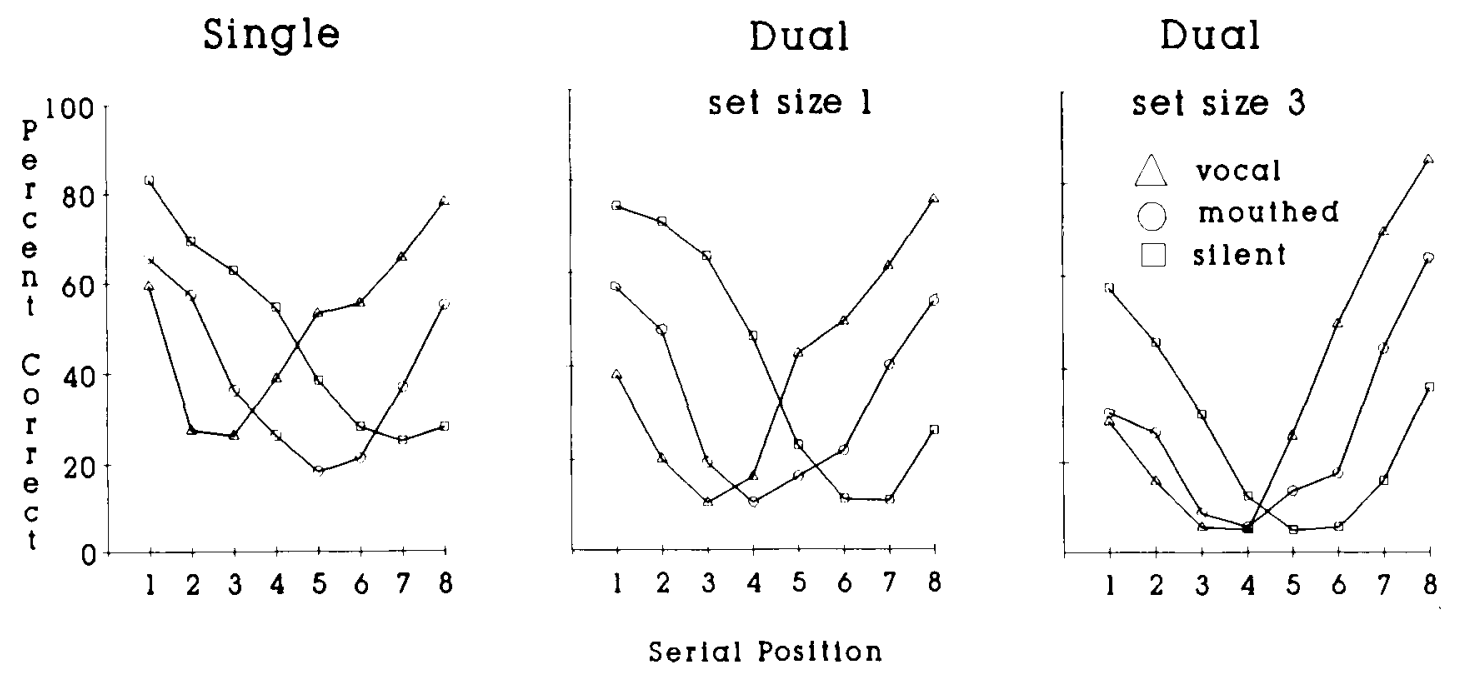

Figure 3. Percent of correctly recalled items for subjects whose performance on the first two serial positions is above $15 \%$. 
emphasis on performing one of the tasks. Rather than abandon recall, subjects may deemphasize the digit search task. This pattern would be particularly evident towards the end of a dual-task trial, since more and more words need to be remembered as one simultaneously searches for digits. Thus, in our recall analyses, one possible explanation for the stability of the modality effect during the dual task is that the subjects abandoned searching for digits toward the end of trials, and concentrated on remembering words. This switch in attention would be seen not only in higher recency, but in longer reaction times and higher error rates for the search task towards the end of dual-task trials. In addition, reaction times and errors for the final stimulus frames should be greater on dualtask trials than for corresponding frames of single search task trials where there are no to-be-remembered words. As will be demonstrated below, these predictions were not supported by either the reaction time or error rate analysis.

\section{Visual Search Task}

Due to technical difficulties, reaction times and error rates for 1 subject in each of the modality conditions were discarded. Reaction times were measured from the onset of a stimulus frame to the subject's initiation of a response. The reaction time analysis reflects the mean median response times for each subject on frames in which a correct positive or negative response was made. The error analysis was computed on the total number of errors committed. These included frames to which subjects failed to respond within the 2-sec frame time allotted. However, such failures to respond were rare (approximately $3 \%$ of all trials). The reaction time and error measures were independently submitted to a 3 (modality) $\times 2$ (day) $\times$ 2 (yes $/$ no) $\times 2$ (task) $\times 2$ (level of difficulty) $\times 8$ (serial position) mixed ANOVA reflecting the between-subjects factor of modality (mouthed, vocalized, or silently read words), and the within-subjects factors of day (Day 2 or 3 of testing), yes/no (whether correct responses were positive [yes] or negative [no]), task (single visual search or dual task), level of difficulty (whether subjects were required to search with a memory-set size of one or three items) and serial position (Stimulus Frames 1-8).

Reaction times. If the stability of the modality effect is due to subjects' switching their attention from searching for digits to remembering words, then in the dual task, reaction times should be greater for the final serial position than for preterminal positions, and they should contrast with more consistent performance across serial positions in the single task where subjects do not have to remember words. However, as shown in Figure 4 (collapsing across positive and negative responses), reaction times remained stable across Serial Positions 3 to 8 for both tasks, and for all modalities. This finding suggests that the subjects maintained their attention to the visual search task even when simultaneously attempting to remember words. This conclusion was supported by the lack of an interaction of task $\times$ serial position $[F(7,168)=$ $\left.1.41, M S_{e}=13,918.50\right]$ or modality $\times$ task $\times$ serial position $\left[F(14,168)=1.31, M S_{\mathrm{c}}=13,918.50\right]$. Although there was a significant effect of task $\times$ serial position $\times$ level of difficulty $\left[F(7,168)=3.22, M S_{\mathrm{e}}=12,785.28\right]$, this interaction was confined to the second serial position, and did not interact with modality $(F<1)$. Similar results were obtained regardless of whether the correct search response was positive or negative. There was no interaction of yes $/$ no $\times$ task $\times$ serial position $[F(7,168)=1.09$, $\left.M S_{\mathrm{e}}=12,337.52\right]$, or of modality $\times$ yes $/$ no $\times$ task $\times$ serial position $\left[F(14,168)=1.10, M S_{\mathrm{e}}=12,337.52\right]$. The reaction time analysis supports the notion that the modality effect obtained in recall under concurrent load conditions cannot be attributed to subjects' switching their attention from the search task to remembering words.

One interpretation of these results is that the modality effect does not require resources from a limited-capacity system. An alternative explanation is that the search task rather than the recall task is not resource-demanding. One way to examine whether a task demands resources is to manipulate its difficulty. If performance reflects a limited capacity, then increasing the requirements of the task should result in greater resource demands and subsequent decrements in performance. Support for the notion that the search task was resource-demanding can be found in the effect of level of difficulty $\left[F(1,24)=211.56, M S_{e}\right.$ $=200,818.19]$, indicating that the subjects searched faster with a memory-set size of one than with a memory-set size of three. Converging evidence comes from the main effect of task $\left[F(1,24)=73.54, M S_{\mathrm{e}}=227,097.59\right]$, indicating significantly higher reaction times during the dual task than during the single task. Because the recall analysis also showed performance decrements as a function of concurrent load, these results support the contention that searching for digits while attempting to remember a series of words requires cognitive resources from the same limited pool. But as the recall analyses demonstrated, this system does not appear to mediate modality effects.

There were several other statistically significant results obtained from the reaction time analysis. None of these effects can be used to explain the modality effect, since they did not correspond to differences in recency, nor do they demonstrate interactions of recency and task type, or of these factors with modality of presentation. The results showed that, overall, correct positive responses were faster than negative ones $\left[F(1,24)=54.35, M S_{\mathrm{e}}=\right.$ 204,222.11], and that there was a significant effect of serial position $\left[F(7,168)=17.83, M S_{\mathrm{e}}=25,765.20\right]$. The significant interactions comprised yes/no $\times$ level of difficulty $\left[F(1,24)=29.44, M S_{\mathrm{e}}=65,230.61\right]$, yes/no $\times$ serial position $\left[F(7,168)=6.34, M S_{\mathrm{e}}=13,637.33\right]$, level of difficulty $\times$ serial position $[F(7,168)=6.48$, $\left.M S_{\mathrm{e}}=14,877.73\right]$, and modality $\times$ yes $/$ no $\times$ serial position $\left[F(14,168)=2.06, M S_{e}=13,637.33\right]$.

The results of the reaction time analysis support the preliminary conclusions drawn from the recall data. Search time in the dual task remained relatively constant across modalities and serial positions, suggesting that mo- 
$\triangle$ vocal $\quad$ mouthed $\quad \square$ silent
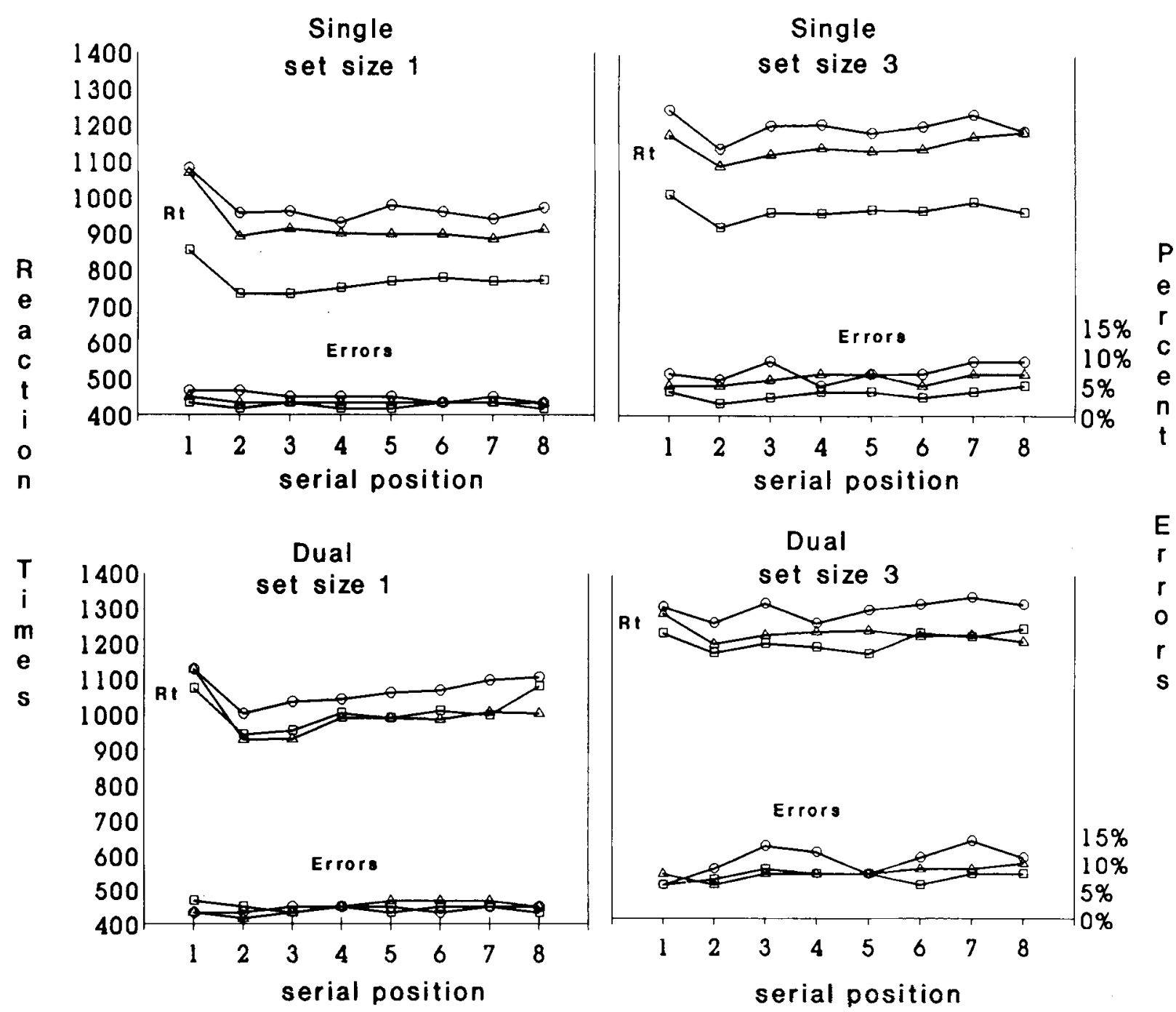

Figure 4. Mean median reaction times and percent errors (collapsing across positive and negative trials) as a function of modality of presentation, serial position, and whether search performance reflects the single or dual task.

dality effects obtained under concurrent load were not due to subjects' switching attention. In addition, performance in both the search and recall tasks declined with concurrent load, indicating that performing these tasks simultaneously requires resources from the same source. In contrast, the magnitude of the modality effect was not influenced by the demands of a conccurrent task, regardless of whether subjects were vocalizing or mouthing words. Taken together, these findings support the contention of the PAS model that modality effects are associated with both gestural and auditory stimulation, and are not mediated by an attention-demanding mechanism. However, evidence for selective attention can be found not only in changes in reaction times, but also in the number of errors that subjects commit. Although speed may remain relatively stable, the degree to which attention is allocated to a task can also be assessed by examining the accuracy with which subjects can perform.

Errors. The results of the error analysis can be seen in the lower half of Figure 4. Overall, errors were low, around $10 \%$. Errors in the final frames of stimulus lists remained relatively stable across tasks and modalities, supporting the contention that the modality effects obtained with the recall data are not a function of switches in attention towards the end of dual-task trials. Thus, although there was a task $\times$ serial position effect $[F(7,168)$ $=2.49, M S_{\mathrm{e}}=0.62 \mathrm{]}$, post hoc tests indicated that interactive effects were confined to primacy positions, as was the interaction of modality $\times$ task $\times$ serial position $\left[F(14,168)=1.81, M S_{e}=0.62\right]$. In addition, there was further evidence that the visual search task was resourcedemanding, since performance declined as level of dif- 
ficulty increased $\left[F(1,24)=223.19, M S_{\mathrm{e}}=2.46\right]$, and there was better performance in the single than in the dual task $\left[F(1,24)=17.04, M S_{\mathrm{e}}=3.88\right]$.

As with the reaction times analysis, several other significant effects were obtained. However, none of these effects can be used to explain the modality effect, since they did not correspond to differences in recency, nor do they demonstrate interactions of recency and task type, or of these factors with modality of presentation. The error analysis showed more errors on Day 2 than on Day 3 of testing $\left[F(1,24)=7.46, M S_{\mathrm{e}}=1.46\right]$, and significant effects were associated with modality $\times$ level of difficulty $\left[F(2,24)=5.47, M S_{\mathrm{e}}=2.46\right]$, yes $/$ no $\times$ level of difficulty $\left[F(1,24)=42.77, M S_{\mathrm{e}}=1.16\right]$, yes $/$ no $\times$ serial position $\left[F(7,168)=13.74, M S_{\mathrm{e}}=0.77\right]$, yes $/$ no $\times$ task $\times$ level of difficulty $\left[F(1,24)=7.28, M S_{\mathrm{e}}=0.43\right]$, and yes/no $\times$ level of difficulty $\times$ serial position $[F(7,168)=$ $\left.16.75, M S_{\mathrm{e}}=0.64\right]$. No other effects in this analysis were statistically significant.

\section{DISCUSSION}

Varied-mapping visual search tasks are commonly used to measure attention, since the speed and accuracy with which subjects search for items appear to reflect the amount of processing dedicated to the task. If two tasks can be performed concurrently, with neither showing a substantial decline in performance, they presumably do not rely on the same resources (Fisk, Derrick, \& Schneider, 1986-1987). This pattern of behavior would be expected, for example, when one task simply does not require attention. This is a fundamental characteristic of an automatic process, in that it is not limited by shortterm or working memory (Schneider \& Shiffrin, 1977; Shiffrin \& Schneider, 1977). In the typical dual-task procedure, performance on the automatically activated task is not reduced by increasing the difficulty of the attention-demanding concurrent task.

Before concluding that the modality effect reflects an automatic process, it was necessary to demonstrate that the concurrent visual search task indeed demanded resources. If performance reflects a limited-capacity system, then increasing the requirements of the task should result in greater attentional demands and performance decrements. In both the reaction time and the error analyses, performance declined when the subjects had to search for three memory-set items rather than one. These results indicate that the search task was capacitydemanding. Furthermore, these increases in reaction time and errors corresponded to overall decreases in recall, suggesting that performance on the search and recall tasks relies on the same limited resource pool.

It also is necessary to show that subjects did not switch their attention during the dual task from searching for digits to remembering words. If responses to the final search frames of the dual task were longer or more error prone than in the single visual search task, then any ef- fects associated with recency could be attributed to an attention switch. Again, the reaction time and error measures show consistent and accurate responding in final test frames across tasks and modalities. Given these results, it is appropriate to examine how performing the visual search task influenced the modality effect.

The recall analysis showed clear modality effects associated with both mouthing and vocalizing in the single task, thereby replicating previous findings with mouthed and vocalized stimuli (Greene \& Crowder, 1984; Nairne $\&$ Walters, 1983). More importantly, our study showed that the magnitude of these modality effects was not reduced by the presentation of an attention-demanding concurrent task. The recall analysis on the final serial position indicated that the relationship between vocalizing and silent reading, mouthing and silent reading, and vocalizing and mouthing was not altered, regardless of whether subjects were simply remembering words or were remembering words while simultaneously searching for digits. As is characteristic of an automatic process, the modality effects associated with both mouthing and audition do not appear to be limited by working-memory capacity. As predicted by the PAS model of echoic memory, these effects appear to reflect a preattentive mechanism.

One other interesting finding arose from the recall measure. With all modalities of presentation, recency increased when task demands increased, and when primacy decreased. Since the reaction time and error measurements for the dual task did not show a primacy-recency tradeoff, we suggest that the changes in the serial position functions associated with recall reflect the strategies that subjects employed to remember words while simultaneously attempting to maintain a consistent level of search performance. In a dual-task trial, it is critical that subjects attend to the memory-set items, because these items are used in the subsequent digit search. It is possible that when digits in the memory set were initially presented, the subjects allocated resources to trying to remember them. This would affect the encoding of words that were presented at the same time. Of course, these would be words presented at the beginning of a trial-that is, in primacy positions. As a result, primacy decreased as concurrent load increased. Why, then, does recency improve? We present two possible explanations: One reason may be that once the search digits are memorized, subjects are able to allocate more resources to the words. Because there are fewer of the primacy items that have been stored, recency items have a greater probability of being remembered. That is, the attentional resources that would otherwise have been allocated to primacy items are now used to store final list items. Another reason may be that because primacy items were not well encoded, they are no longer a source of output interference. With this interference reduced, recency items are more likely to be retrieved. Our study was not designed to distinguish between these possibilities. It is important to emphasize, however, that although the serial position functions associated with recall 
were influenced by the concurrent task, it was shown that neither a tradeoff explanation, nor an explanation based on practice effects can be used to explain the stability of the modality effect. Although serial position functions may index a limited-capacity processor, the modality effect does not appear to reflect such limitations.

Although we have argued that our findings support an echoic memory model, several other models have been proposed to explain modality effects. While some of these accounts, such as those based on primary linguistic coding (Shand \& Klima, 1981) or the static versus changing state of the stimulus material (Campbell \& Dodd, 1980) have not held up under empirical scrutiny (see Penney, 1989 , for a review), others are so recent that their worth is not yet known. We present two such models, and examine the extent to which each can handle our data.

Perhaps the greatest challenge to an echoic memory explanation of modality effects has been reported by Glenberg and his colleagues (Glenberg, 1984; Glenberg \& Swanson, 1986), who found long-term modality effects, even when there were several seconds of interitem auditory and visual distractor activity, and similar activity between the final stimulus items and recall. Any model that attributes modality effects to a brief echo that is sensitive to subsequent auditory interference cannot account for these long-term effects. As an alternative, Glenberg (1984; Glenberg \& Swanson, 1986) argued against sensory storage theories, suggesting that recency and modality effects reflect a general retrieval process based on temporal coding. During encoding, there is a defining of time-based search sets that differ in the number of items represented. The more items included in a single set, the less likely that any particular item of that set will be recalled. In general, recency results because the final item of verbal lists is included in a small search set relative to earlier list items. The difference between modalities is posited to reflect the structure of the cognitive system such that auditory presentation leads to a more finely grained encoding of time than does visual presentation. Supporting this view, Glenberg and Swanson (1986) showed that increasing the interval between initial and final list items increases recency, presumably because lengthening the time between these items results in a more temporally distinct final search set.

But there are findings in our study that are not easily explained by the temporal distinctiveness theory. During the dual task, the visual digit search provided distractor activity, and, although we increased overall working memory load, interitem distractor time remained constant across the levels of the dual task. Without manipulating the temporal interval between list items, we obtained substantial changes in recency for all modalities.

Although the notion of reduced output interference during retrieval, or increases in resources during encoding, could be used to account for our overall improvements in recency, it is not clear how the temporal distinctiveness model would predict that these processes would effect silently read lists differently than mouthed or vocal- ized lists. Glenberg and Swanson argued that spoken input leads to a more finely grained encoding of time than does silent reading, but recall is posited to reflect the same retrieval processes for all modalities. Therefore, if there is a tradeoff between primacy and recency, the same pattern should be demonstrated across modalities. Not only should recency increase as primacy decreases, but all subjects who show high primacy should also show less recency. When the data for subjects with high primacy were reanalyzed, only the silent reading condition showed smaller final item performance, producing a pattern that was different from vocalized and mouthed lists.

We have argued that the PAS model best accommodates our data, because, within this framework, echoic memory is an automatically activated and preattentive process. There is, however, another model of echoic memory, in which auditory and visual processes are posited to reflect separate "streams" in the cognitive system (Penney, 1989). Accordingly, there is an echoic trace, termed the acoustic (A) code, that is automatically activated by audition, is sensitive to subsequent auditory masks, and is limited in duration and capacity (although the estimates of these parameters are somewhat larger than they are for PAS). As with PAS, it is the echoic trace of the A code that produces superior recency for spoken lists. A phonological code is also automatically generated by audition, but unlike the echoic trace, this code requires attention to maintain.

One problem with the separate-streams model is the assumption that effects produced by silent mouthing do not reflect the same processes as those found with speech. As noted earlier, there are smaller modality effects associated with mouthing, and this condition is not consistently influenced by phonemic similarity. Penney (1989) suggested that one possibility is that mouthing may produce a phonological code similar to audition, but not an A code. We found, however, that performance on the final item of mouthed list is is not influenced by the attentional demands of a concurrent task, a property attributable to Penney's A code.

Another difficulty with the separate-streams model is that auditory and visual processes in short-term memory are thought to be qualitatively different, relying on different pools of resources. Again our data speaks to this issue by showing that performing a visual search while attempting to remember mouthed or vocalized words produces decrements in both tasks. These results support the notion that there is some competition for resources between modalities. While there may be qualitatively different codes across modalities, maintenance of codes that require attention seem to be limited by a common mechanism.

In conclusion, the results of our study indicate that modality effects are mediated by a preattentive process specialized for auditory input and silent gestures associated with speech. These findings clearly support the PAS model. In the temporal distinctiveness theory, assumptions about recency are critical in explaining why the modality effect is confined to terminal list items. Because 
this model cannot account for our recency effects, they are also insufficient to explain our modality effects. In addition, although the separate-streams model does predict that modality effects reflect preattentive processes, other predictions such as separate pools of resources for auditory and visual information processing and separate codes for mouthed versus vocalized input were not supported by our data.

\section{REFERENCES}

Atkinson, R. C., \& Shiffrin, R. M. (1968). Human memory: A proposed system and its control processes. In K. W. Spence \& J. T. Spence (Eds.), The psychology of learning and motivation. New York: Academic Press.

Balota, D. A., ENGle, R.W. (1981). Structural or strategic figures in the stimulus suffix effect. Journal of Verbal Learning \& Verbal Behavior, 20, 346-357.

CAMPBELL, R., DoDD, B. (1980). Hearing by eye. Quarterly Journal of Experimental Psychology, 32, 85-99

Conrad, R., Hull, A. J. (1968). Input modality and the serial position curve in short-term memory. Psychonomic Science, 10, 135-136.

Corballis, M. C. (1966). Rehearsal and decay in immediate recall of visually and aurally presented items. Canadian Journal of Psychology, 20, 43-51.

CrowDER, R. G. (1971). The sounds of vowels and consonants in immediate memory. Journal of Verbal Learning \& Verbal Behavior, 10. 587-596.

Crowder, R. G. (1973). The delayed stimulus suffix effect following arhythmic stimulus presentation. Quarterly Journal of Experimental Psychology, 25, 433-439.

Crowder, R. G. (1983). The purity of auditory memory. Philosophical Transactions of the Royal Society of London, B302, 251-265.

Crowder, R. G., \& Morton, J. (1969). Precategorical acoustic storage (PAS). Perception \& Psychophysics, 5, 365-373.

Fisk, A. D., DerRick, W. L., SChneider, W. (1986-87). A methodological assessment and evaluation of dual-task paradigms. Current Psychological Research \& Reviews, 5, 315-327.

FranCIS, W. N., \& KUČERA, H. (1982). Frequency analysis of english language. Boston, MA: Houghton Mifflin.

GlenBerg, A. M. (1984). A retrieval account of the long-term modality effect. Journal of Experimental Psychology: Learning, Memory. \& Cognition, 10, 16-31.

GlenberG, A. M., \& Swanson, N. G. (1986). A temporal distinctiveness theory of recency and modality effects. Joumal of Experimental Psychology: Learning, Memory, \& Cognition, 12, 3-15.
GreenberG, S. N. \& ENGLE, R. W. (1983). Voice change in the stimulus suffix effect: Are the effects structural or strategic? Memory \& Cognition, 11, 551-556.

Greene, R. L., Crowder, R. G. (1984). Modality and suffix effects in the absence of auditory stumulation. Joumal of Verthal Leaming \& Verbal Behavior, 13, 430-447.

Greene, R. L., C Crowder, R. G. (1986). Recency effects in delayed recall of mouthed stimuli. Memory \& Cognition, 14, 355-360.

Morton, J., Crowder, R. G., \& Prussin, H. A. (1971). Experiments with the stimulus suffix effect. Journal of Experimental Psychology, 91, 169-190.

MURRAY, D. J. (1966). Vocalization-at-presentation, auditory presentation and immediate recall, with varying recall methods. Quarterly Journal of Experimental Psychology, 18, 9-18.

Nairne, J. S. \& Crowder, R. G. (1982). On the locus of the stimulus suffix effect. Memory \& Cognition, 10, 350-357.

NAIRNE, J. S., \& Waters, J. V. (1983). Silent mouthing produces modality-and suffix-like effects. Journal of Verbal Leaming \& Verbal Behavior, 22, 475-483.

NeIsser, U. (1967). Cognitive psychology. Englewood Cliffs, NJ: Prentice-Hall.

Penney, C. G. (1989). Modality effects and the structure of short-term verbal memory. Memory \& Cognition, 17, 398-422.

SChneider, W., Shiffrin, R. M. (1977). Controlled \& automatic human information processing: I. Detection, search, and attention. Psychological Review, 84, $1-66$

Shand, M. A., KLimA, E. S. (1981). Nonauditory suffix effects in congenitally deaf signers of American Sign Language. Journal of Experimental Psychology: Human Learning \& Memory, 7, 464-474.

Shiffrin, R. M., SChNeider, W. (1977). Controlled \& automatic human information processing: II. Perceptual learning, automatic attending, and a general theory. Psychological Review, 84, 127-185.

SPOEHR, K. T. Corin, W. J. (1978). The stimulus suffix effect as a memory coding phenomenon. Memory \& Cognition, 6, 583-589.

Turner, M. L., La Pointe, L. B., Cantor, J., Reeves, C. H., GRIFFETH, R. H., ENGLE, R. W. (1987). Recency with suffix effects found with auditory presentation and with mouthed visual presentation: They are not the same thing. Journal of Memory \& Language, 26, 138-164.

Watkins, M. J., Watkins, O. C., \& Crowder, R. G. (1974). The modality effect in free and serial recall as a function of phonological similarity. Journal of Verbal Learning \& Verbal Behavior, 13, 430-447.

(Manuscript received September 15, 1988; revision accepted for publication March 27, 1989.) 